\title{
Unconventional Disorder Effects in Correlated Superconductors
}

\author{
Maria N. Gastiasoro, ${ }^{1}$ Fabio Bernardini, ${ }^{2}$ and Brian M. Andersen ${ }^{1}$ \\ ${ }^{1}$ Niels Bohr Institute, University of Copenhagen, Juliane Maries Vej 30, 2100 Copenhagen, Denmark \\ ${ }^{2}$ CNR-IOM-Cagliari and Dipartimento di Fisica, Università di Cagliari, 09042 Monserrato, Italy
}

(Received 18 July 2016; published 15 December 2016)

\begin{abstract}
We study the effects of disorder on unconventional superconductors in the presence of correlations, and explore a novel correlated disorder paradigm dominated by strong deviations from standard AbrikosovGor'kov theory due to generation of local bound states and cooperative impurity behavior driven by Coulomb interactions. Specifically we explain under which circumstances magnetic disorder acts as a strong poison destroying high- $T_{c}$ superconductivity at the sub-1\% level, and when nonmagnetic disorder, counterintuitively, hardly affects the unconventional superconducting state while concomitantly inducing an inhomogeneous full-volume magnetic phase. Recent experimental studies of Fe-based superconductors have discovered that such unusual disorder behavior seems to be indeed present in those systems.
\end{abstract}

DOI: 10.1103/PhysRevLett.117.257002

The study of disorder effects in the presence of electronic interactions currently constitutes a very active line of research. For unconventional superconductors this is motivated largely by the fact that these systems are made superconducting by "chemical disordering" (charge doping), but also boosted by controversies of the correct microscopic model, and a rapid development of local experimental probes [1-3]. Focusing on multiband $\mathrm{Fe}-$ based superconductors (FeSC), disorder studies have proven exceptionally rich and strongly material dependent [4]. Scanning tunneling spectroscopy found a plethora of exotic atomic-sized impurity-generated states [5-8], NMR and neutrons observed evidence of glassy magnetic behavior $[9,10]$, and $\mu \mathrm{SR}$ discovered magnetic phases generated by nonmagnetic disorder [11,12]. The origin and nature of the resulting inhomogeneous phases and their properties in terms of thermodynamics and transport constitute an important open problem in the field.

Here, we present a theoretical study of correlation-driven emergent impurity behavior of both magnetic and nonmagnetic disorder in unconventional multiband superconductors [13-18]. For the case of magnetic disorder, we find that correlations antiscreen the bare impurity potential, and significantly enhance interimpurity Ruderman-KittelKasuya-Yosida (RKKY) interactions by inducing nonlocal long-range magnetic order which operates as an additional competitor to superconductivity. This results in aggressive $T_{c}$-suppression rates where superconductivity is wiped out by sub-1\% concentrations of disorder well beyond the behavior obtained within Abrikosov-Gor'kov (AG) theory [19-21]. By contrast, for weak nonmagnetic disorder the correlated unconventional superconducting state is largely immune to disorder, in agreement with earlier one-band studies, finding that correlations enhance the screening of disorder potentials and thereby reduce pair-breaking and scattering rates compared to the noninteracting case [22-27]. As we show, however, in the current multiband case, additional impurity-generated bound states play an important role in supporting $T_{c}$. This resilience to nonmagnetic disorder is remarkable since favorable clusters of impurities locally pin magnetic order, eventually causing a volume-full inhomogeneous magnetic state which coexists with superconductivity. These theoretical results are in excellent agreement with extensive experimental studies of Mn- and Ru-substituted 1111 FeSCs.

Model.--Interactions are included by the standard multiorbital Hubbard term [28,29]

$$
\begin{aligned}
\mathcal{H}_{\mathrm{int}}= & U \sum_{\mathbf{i}, \mu} \hat{n}_{\mathbf{i} \mu \uparrow} \hat{n}_{\mathbf{i} \mu \downarrow}+\left(U^{\prime}-\frac{J}{2}\right) \sum_{\mathbf{i}, \mu<\nu, \sigma \sigma^{\prime}} \hat{n}_{\mathbf{i} \mu \sigma} \hat{n}_{\mathbf{i} \nu \sigma^{\prime}} \\
& -2 J \sum_{\mathbf{i}, \mu<\nu} \vec{S}_{\mathbf{i} \mu} \cdot \vec{S}_{\mathbf{i} \nu}+J^{\prime} \sum_{\mathbf{i}, \mu<\nu, \sigma} \hat{c}_{\mathbf{i} \mu \sigma}^{\dagger} \hat{c}_{\mathbf{i} \mu \bar{\sigma}}^{\dagger} \hat{c}_{\mathbf{i} \nu \bar{\sigma}} \hat{c}_{\mathbf{i} \nu \sigma},
\end{aligned}
$$

where $\mu, \nu$ are orbital indices, $\mathbf{i}$ denotes lattice sites, and $\sigma$ is the spin. The interaction includes intraorbital (interorbital) repulsion $U\left(U^{\prime}\right)$, the Hund's coupling $J$, and the pair hopping energy $J^{\prime}$. We assume $U^{\prime}=U-2 J$ and $J^{\prime}=J$ and fix $J=U / 4$. Nonmagnetic and magnetic disorder give rise to the terms $\mathcal{H}_{\text {imp }}=\sum_{\left\{\mathbf{i}^{*}\right\} \mu \sigma} V_{\mu} \hat{c}_{\mathbf{i}^{*} \mu \sigma}^{\dagger} \hat{c}_{\mathbf{i}^{*} \mu \sigma}$ and $\mathcal{H}_{\text {imp }}=I \sum_{\left\{\mathbf{i}^{*}\right\} \mu \sigma} \sigma S_{\mu} \hat{c}_{\mathbf{i}^{*} \mu \sigma}^{\dagger} \hat{\sigma}_{\mathbf{i}^{*} \mu \sigma}$, respectively. Here $V_{\mu}\left(S_{\mu}\right)$ denotes the impurity potential (magnetic moment) in orbital $\mu$ at the disorder sites given by the set $\left\{\mathbf{i}^{*}\right\}$ coupled to the charge (spin) density of the itinerant electrons. We focus on FeSC and use a five-band model

$$
\mathcal{H}_{0}=\sum_{\mathbf{i} \mathbf{j}, \mu \nu, \sigma} t_{\mathbf{i} \mathbf{j}}^{\mu \nu} \hat{c}_{\mathbf{i} \mu \sigma}^{\dagger} \hat{c}_{\mathbf{j} \nu \sigma}-\mu_{0} \sum_{\mathbf{i} \mu \sigma} \hat{n}_{\mathbf{i} \mu \sigma},
$$

with tight-binding parameters appropriate for 1111 pnictides [30]. The model $H_{0}+H_{\text {int }}$ exhibits a transition to a bulk $(\pi, 0)$ spin density wave phase at a critical value $U_{c}=0.89 \mathrm{eV}$. We parametrize the interactions in terms of 


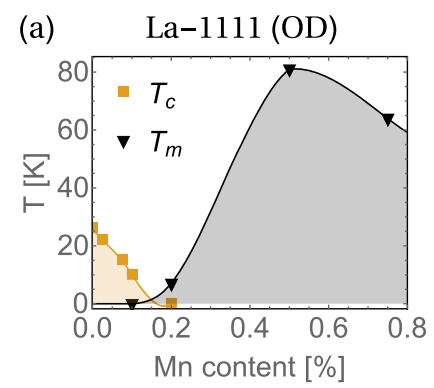

(c)

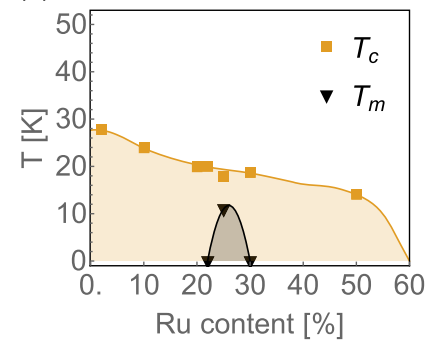

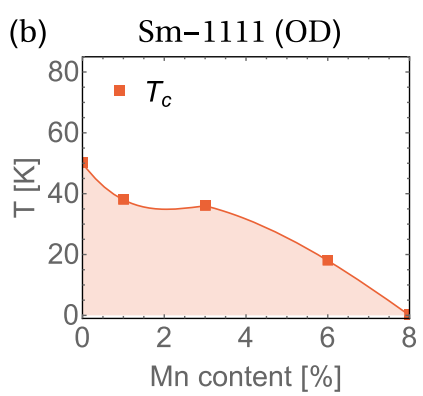

(d)

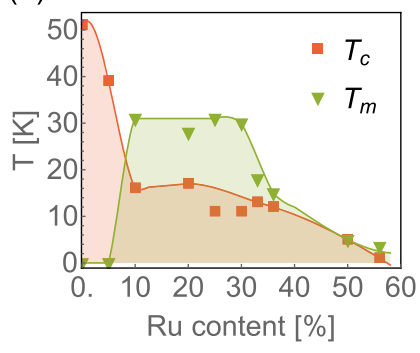

FIG. 1. Experimentally obtained superconducting $T_{c}$ and magnetic $T_{m}$ transition temperatures in OD La-1111 (a),(c) and Sm-1111 (b),(d) vs magnetic disorder (a),(b) and nonmagnetic disorder (c),(d). The data were adapted from Refs. [11,12,41,42,44].

$u=U / U_{c}$, and operate in the regime $u<1$. Superconductivity is included by $\mathcal{H}_{\mathrm{BCS}}=-\sum_{\mathbf{i} \neq \mathbf{j}, \mu \nu}\left[\Delta_{\mathbf{i} \mathbf{j}}^{\mu \nu} \hat{c}_{\mathbf{i} \mu \uparrow}^{\dagger} \hat{c}_{\mathbf{j} \nu \downarrow}^{\dagger}+\right.$ H.c.], with $\Delta_{\mathbf{i j}}^{\mu \nu}=\sum_{\alpha \beta} \Gamma_{\mu \alpha}^{\beta \nu}\left(\mathbf{r}_{\mathbf{i j}}\right)\left\langle\hat{c}_{\mathbf{j} \beta \downarrow} \hat{c}_{\mathbf{i} \alpha \uparrow}\right\rangle$ being the superconducting order parameter, and $\Gamma_{\mu \alpha}^{\beta \nu}\left(\mathbf{r}_{\mathbf{i j}}\right)$ denoting the Fourier transform of the effective pairing vertex [17]. In agreement with a general $s \pm$ pairing state in FeSC, we include next-nearest neighbor (NNN) intraorbital pairing. For further computational details and parameter dependence, we refer to the Supplementary Material (SM) [31].

Magnetic disorder.-The study of magnetic disorder is motivated largely by the following experimental facts summarized in Figs. 1(a) and 1(b): In optimally doped (OD) $\mathrm{LaFeAsO}_{1-x} \mathrm{~F}_{x}$ (La-1111) with $T_{c} \sim 30 \mathrm{~K}$ a mere $\sim 0.2 \%$ magnetic $\mathrm{Mn}$ ions is enough to destroy the superconducting state [41]. This extreme destruction rate of bulk superconductivity has been recently dubbed "the poisoning effect" [42]. Interestingly, immediately beyond $\sim 0.2 \%$, the same minute amount of $\mathrm{Mn}$ ions generate a static magnetic phase with full volume fraction and sizable magnetic transition temperature $T_{m}$ defined as the highest $T$ with a $50 \%$ magnetic volume fraction as seen by $\mu \mathrm{SR}$. Recently it was found that this magnetic phase is $(\pi, 0)$ ordered with a concomitant orthorhombic structural transition similar to the undoped system [43]. By contrast, for OD $\mathrm{SmFeAsO}_{1-x} \mathrm{~F}_{x}(\mathrm{Sm}-1111)$, the corresponding $T_{c}$-suppression rate is much slower with $\sim 8 \%$ of $\mathrm{Mn}$ required to destroy superconductivity [44] [Fig. 1(b)].

Figure 2(a) shows the suppression of $T_{c}$ as a function of magnetic impurity concentration obtained within our model. Without correlations $(u=0)$ the $T_{c}$ suppression

(a)

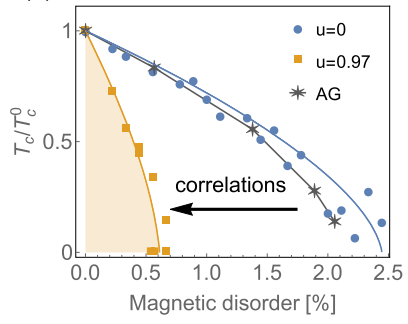

(b)

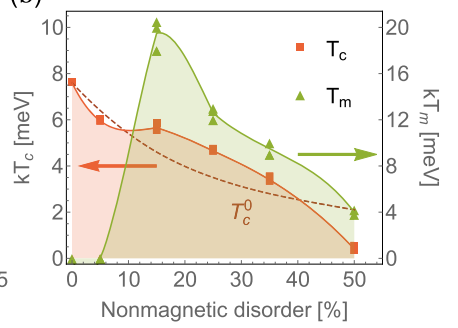

FIG. 2. Superconducting critical temperature $T_{c}$ vs magnetic (a) and nonmagnetic (b) impurity concentrations. (a) Effect of electronic correlations on $T_{c} / T_{c}^{0} \quad\left(T_{c}^{0}=3.6 \mathrm{meV}\right)$, compare $u=0$ and $u=0.97$, for a case with orbital independent impurities modeled by $I S_{\mu}=0.38 \mathrm{eV}$. The $u=0$ curve agrees with standard AG theory (gray stars). (b) $T_{c}$ (red squares) and magnetic critical temperature $T_{m}$ (green triangles) induced by nonmagnetic disorder. Dashed curve in (b) shows $T_{c}$ for the clean system $T_{c}^{0}$ where only a band-widening effect has been included (i.e. no disorder) as discussed in the main text.

follows the curve expected from AG theory. The main result of Fig. 2(a) is the much faster $T_{c}$-suppression rate when including Coulomb interactions. The origin of this strongly modified suppression rate, which appears at odds with the expectation that correlations screen disorder and limit their damaging effect [22-24,26,27], has contributions from both the local scale (immediate vicinity of the impurity sites) and nonlocal scale (interimpurity regions). This is because magnetic impurity moments induce spin polarizations of the surrounding itinerant electrons $m_{\mathbf{i} \mu}$, which renormalize the exchange coupling such that $\tilde{H}_{\mathrm{imp}}=\tilde{I} \sum_{\mathbf{i} \mu \sigma} \sigma \tilde{S}_{\mathbf{i} \mu} \hat{c}_{\mathbf{i} \mu \sigma}^{\dagger} \hat{c}_{\mathbf{i} \mu \sigma}$, where

$$
\begin{aligned}
\tilde{I} \tilde{S}_{\mathbf{i} \mu}= & {\left[I S_{\mu} \delta_{\mathbf{i i}^{*}}-\frac{1}{2}\left(U m_{\mathbf{i} \mu}+J \sum_{\nu \neq \mu} m_{\mathbf{i} \nu}\right)\right] } \\
& \equiv\left[I S_{\mu} \delta_{\mathbf{i i}^{*}}+I_{\mathrm{ind}} S_{\mathbf{i} \mu}\right]
\end{aligned}
$$

is the emergent extended magnetic impurity potential (see Supplemental Material [31] for more details) generated by the induced part, $I_{\text {ind }} S_{\mathbf{i} \mu}$. Note that in order to compare the role of correlations in systems with the same $T_{c}$ in Fig. 2(a), we have used a $U$-independent pairing vertex.

Focusing first on the local part of the effective potential, a line cut of the induced magnetic potential $I_{\text {ind }} s_{\mathbf{i} \mu}$ through a single impurity as a function of $u$ is shown in Fig 3(a). As seen, the extent and amplitude of the resulting magnetic puddle grows significantly with $u$, and results in a realspace structure illustrated in Fig. 3(b). The renormalized magnetic potential $\tilde{I} \tilde{S}_{\mathbf{i} \mu}$ is significantly enhanced at the impurity site, even exceeding the bare value $I S_{\mu}$ at large $u$, and exhibits sizable antiparallel neighbors. Superconductivity is strongly affected by the additional pair-breaking caused by the enhanced local magnetic potentials, and therefore the suppression of the 
(a) $\quad \mathrm{I}_{\text {ind }} \mathrm{s}_{\mathrm{i} \mu}$

(b)

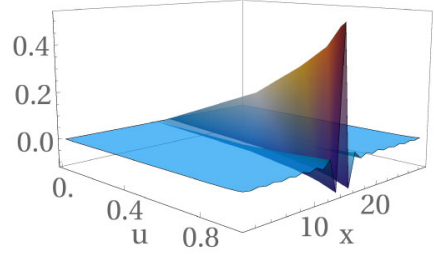

(c) $\quad \Delta_{\mathrm{i} \mu} / \Delta_{0}$

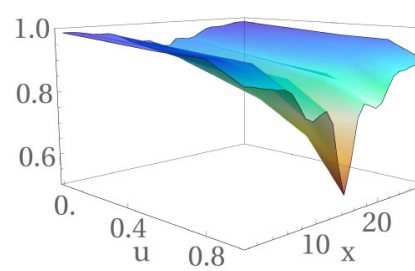

(e)

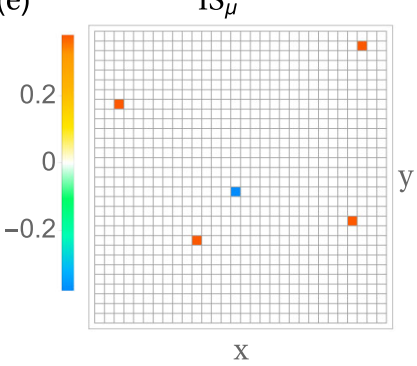

(d) $\quad$ IS ${ }_{\mu}$ local o full
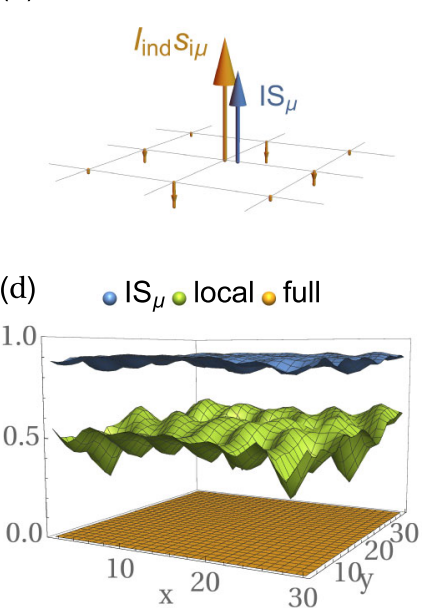

(f)

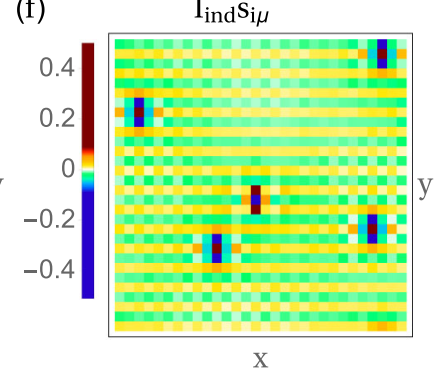

FIG. 3. (a) Induced magnetic potential, $I_{\text {ind }} s_{\mathbf{i} \mu}$, along a cut through a magnetic impurity and as a function of $u$. Correlations renormalize local magnetic potentials as shown in (b) where the orange (blue) arrows show the induced (bare) parts for $u=0.97$. (c) Local suppression of $\Delta_{\mathbf{i} \mu}$ relative to its value in the clean system $\Delta_{\mu}^{0}$ vs $u$. (d) Real-space map of $\Delta_{\mathbf{i} \mu} / \Delta_{\mu}^{0}$ in the presence of $0.55 \%$ magnetic disorder. The blue surface shows the suppression from only the bare moments, i.e. $u=0$, but self-consistently obtained gaps beyond AG theory. Including the local correlationenhanced magnetic moments leads to the green surface, and only by including both local and nonlocal effects is superconductivity fully destroyed (orange). (e),(f) Real-space maps of the $0.55 \%$ (e) bare $I S_{\mu}$ and (f) induced magnetic potential $I_{\mathrm{ind}} S_{\mathbf{i} \mu}$ for $u=0.97$. For all results in this figure, the bare moments are the ones used in Fig. 2 and $\mu=d_{x z}$ orbital.

(bond-averaged, see Supplemental Material [31]) order parameter $\Delta_{\mathbf{i}}$ increases accordingly, as shown in Fig. 3(c). This enhanced local pair-breaking is not, however, the only reason for the enhanced $T_{c}$-suppression rate, which also includes a cooperative (nonlocal) multi-impurity effect.

Indeed, when multiple impurities are included, the correlations among their moments become crucial for lowering the free energy. Specifically, the spin polarized clouds around the impurities prefer to constructively interfere, thereby generating a quasi-long-range ordered magnetic state. [45] The interimpurity regions acquire a resulting finite magnetization due to this enhanced RKKYlike interaction between the impurities. Figures 3(e) and 3(f) compare directly the case in point with $0.55 \%$ uncorrelated disorder $(u=0)$ vs the correlated situation

$(u=0.97)$, respectively. In addition to the local effect discussed above, the system develops $(\pi, 0)$ long-range order magnetization (see also Supplemental Material [31]) in agreement with experiments [43], constituting the additional nonlocal competitor to superconductivity. We show in Fig. 3(d) a plot of these two separate (local vs nonlocal) effects on the suppression of $\Delta_{\mathrm{i}}$. The blue surface is the self-consistent solution of $\Delta_{\mathbf{i}}$ of the $u=0$ system shown in Fig. 3(e). As seen, $\Delta_{\mathbf{i}}$ is hardly affected by the bare magnetic potentials, and this is reflected in the correspondingly low $T_{c}$ suppression of Fig. 2(a). The green surface of Fig. 3(d) shows $\Delta_{\mathbf{i}}$ including the renormalized local potentials, cf. Fig. 3(b). As seen, only when the nonlocal magnetic order is also included, superconductivity is completely wiped out [orange surface in Fig. 3(d)], explaining the aggressive sub-1\% $T_{c}$ suppression rate shown in Fig. 2(a). In the Supplemental Material [31] we discuss the origin of the different $T_{c}$-suppression rate in OD Sm-1111, and show that a critical concentration of $8 \%$ magnetic disorder in that system is consistent with our modeling.

Nonmagnetic disorder.-The study of nonmagnetic disorder is also motivated by a set of puzzling experimental findings from FeSCs summarized in Figs. 1(c) and 1(d), which compare the effect on $T_{c}$ and $T_{m}$ of $\mathrm{Ru}$ ions substituting for $\mathrm{Fe}$ in OD La-1111 and Sm-1111 $[11,12,46] . \mathrm{Ru}$ is isovalent to $\mathrm{Fe}$, and therefore expected to be a source of weak disorder, consistent with the huge amount of $\sim 60 \%$ of $\mathrm{Ru}$ required to suppress $T_{c}$, as seen in Fig. 1(c). An unexpected magnetic phase is induced at intermediate values of $\mathrm{Ru}$ content $x$, centered roughly around $x=0.25$, and existing only at a finite span $\Delta x$ of disorder as seen in Fig. 1(d). The magnetic phase is most pronounced with largest $\Delta x$ and highest $T_{m}$ in Sm-1111 and only marginally present in La-1111, even though this displays the poisoning effect and should be more correlated than Sm-1111. Finally we point out the counterintuitive leveling-off of the $T_{c}$-suppression rate concomitant with the value of Ru content $x_{c}$ where magnetic order sets in, as seen most clearly in the case of Sm-1111 in Fig. 1(d).

In order to capture correctly the effects of large compositional-changing amounts of Ru substitution, it is imperative to include the effect of Ru on the band structure itself. Our first-principles calculations show that the bandwidth roughly doubles with $\mathrm{Ru}$ content going from $x=0$ to $x=1$ in both $\mathrm{LaFe}_{1-x} \mathrm{Ru}_{x} \mathrm{AsO}$ and $\mathrm{SmFe}_{1-x} \mathrm{Ru}_{x} \mathrm{AsO}$ (see Supplemental Material [31] for details). This band-widening effect is accounted for by a renormalization of the hopping amplitudes $t_{\mathbf{i j}}^{\mu \nu} \rightarrow(1+x) t_{\mathbf{i j}}^{\mu \nu}$ in Eq. (2), which changes $T_{c}$ of the clean case as shown by the dashed curve in Fig. 2(b). For concreteness, we focus initially on a case with correlations of intermediate strength, $u=0.7$, since this seems relevant for, e.g., Sm-1111 which exhibits the most pronounced disorder-induced magnetic phase as shown in Fig. 1(d). Consistent with first-principles calculations, [47] we model the random collection of 
nonmagnetic $\mathrm{Ru}$ ions by a set of weak pointlike scatterers with $V_{\mu}=0.03 \mathrm{eV}$ on all orbitals but allow for a phenomenological tuning of the potential on the $d_{3 z^{2}-r^{2}}$ orbital potential $\left(V_{d_{3 z^{2}-r^{2}}}=0.7 \mathrm{eV}\right)$. The latter is required to generate local near-Fermi level LDOS enhancements and concomitant local magnetism for the particular band utilized in this work [30].

Figure 2(b) shows the resulting critical temperatures $T_{c}$ and $T_{m}$ as a function of $x$. As seen, in addition to a much slower $T_{c}$-suppression rate as compared to Fig. 2(a), a magnetic phase centered around $x \sim 25 \%$ is generated above a certain concentration $x_{c}$ of $\mathrm{Ru}$ ions. As a function of $x, T_{c}$ exhibits an initial drop, but, interestingly, the induction of the magnetic phase does not enhance the $T_{c}$-suppression rate as expected from naive competitive considerations, but rather seems to further stabilize superconductivity. The origin for these unconventional disorder effects arise from the emergence of favorable impurity clusters highlighted in Fig. 4(a), which lead to substantial local density of states (LDOS) enhancements of the $d_{3 z^{2}-r^{2}}$ orbital shown in Fig. 4(b). This drives both (i) an induced magnetization as seen from Fig. 4(d) [17,48,49], and (ii) an associated enhancement of the superconducting order parameter $\Delta_{\mathbf{i} d_{3 z^{2}-r^{2}}}$ shown in Fig. 4(f). Through interorbital couplings the boost of $\Delta_{\mathbf{i} d_{3 z^{2}-r^{2}}}$ near the dimers is enough to cause the support for the entire superconducting condensate evident in Fig. 2(b) at intermediate disorder content $\Delta x$, where the enhanced pairing overcompensates the pairbreaking effect of both the disorder and the induced magnetic phase. Interestingly, enhanced pairing from nonmagnetic disorder (As vacancies) was discussed previously for 1111 systems [50,51].

The dimer-induced LDOS enhancement mechanism naturally explains the increase of $T_{m}$ starting at intermediate values of impurity concentration $x_{c} \sim 10 \%$, since no favorable impurity clusters are present below $x_{c}$. As $x$ increases, more dimerlike structures with high LDOS form, and the system eventually acquires a large enough magnetic volume fraction to support a nonzero $T_{m}$. Specifically, $T_{m}$ is defined identically to the experimental $\mu \mathrm{SR}$ definition by the highest $T$ exhibiting a 50\% magnetic volume fraction (see Supplemental Material [31] for details). A site is defined to contribute to the volume fraction if its internal dipolar local field exceeds $|0.5| \mathrm{mT}[11,12]$. In the case of 15\% disorder discussed in Fig. 4 we find a nearly saturated volume fraction as shown in Fig. 4(c), in agreement with experiments [11,12]. From Fig. 4(e), showing the dimer concentration as a function of $x$, one expects a $\max T_{m}$ near $x \sim 40 \%$. However, the band-widening effect $W \rightarrow$ $(1+x) W$ lowers the effective Coulomb correlations, and pushes the magnetic dome to lower $x$. Thus, the position of the induced magnetic dome is a compromise between the dimer-enhanced LDOS and the weakening of correlations due to band widening. The resulting $x$ dependence of (a) Impurity positions

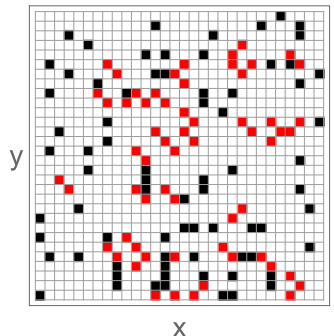

(c)

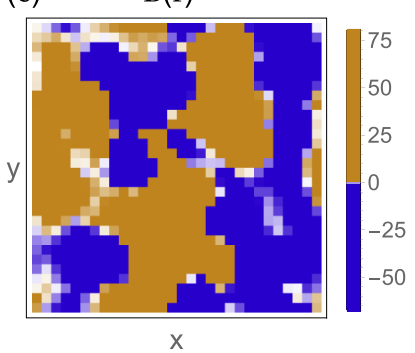

(e)

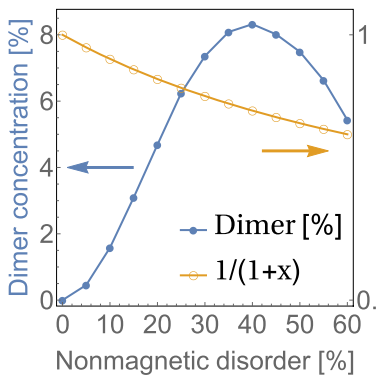

(b) $\quad$ LDOS $_{\mathrm{i} \mu}$

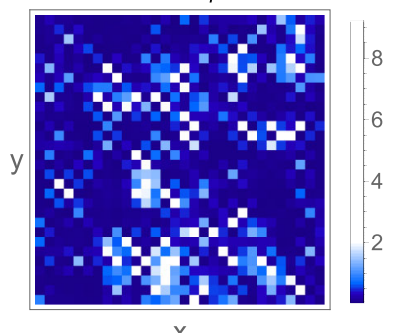

(d)

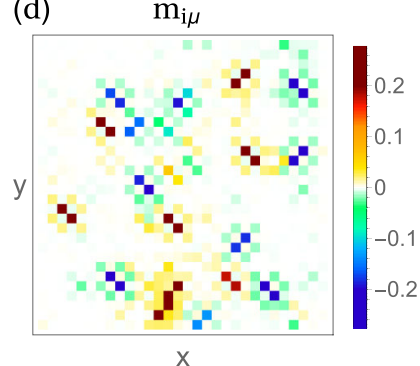

(f) $\quad \Delta_{\mathrm{i} \mu} / \Delta_{0}$

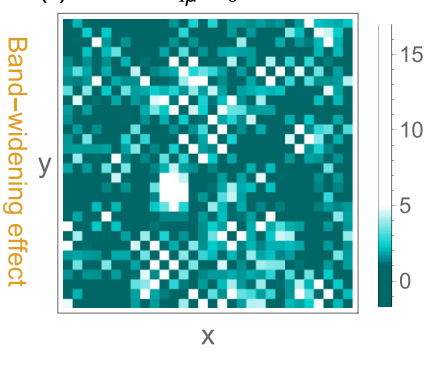

FIG. 4. (a) Positions of a random set of $15 \%$ nonmagnetic disorder. The red tiles highlight favorable dimerlike arrangements, defined by all the impurity sites with an occupied NNN site but not more than one occupied NN site. (b) Real-space map of the LDOS of the $d_{3 z^{2}-r^{2}}$ orbital at $T>T_{c}$ at the Fermi level. (c) Local dipolar field $B(\mathbf{r})=\sum_{\mathbf{i}}\left(m_{\mathbf{i}} /\left|\mathbf{r}_{\mathbf{i}}\right|^{3}\right)$ ( $\mathbf{r}_{\mathbf{i}}$ is the distance between the muon site $\mathbf{r}$ and the moment position $m_{\mathbf{i}}$ of the itinerant electrons) with orange (blue) color indicating regions with field strength larger (smaller) than $0.5 \mathrm{mT}(-0.5 \mathrm{mT})$. (d) Real-space map of the dimer-induced magnetization of the $d_{3 z^{2}-r^{2}}$ orbital. (e) Average dimer concentration (blue dots) and the bandwidth renormalization parameter $1 /(1+x)$ (orange circles) as a function of disorder concentration $x$. (f) Superconducting order parameter of the $d_{3 z^{2}-r^{2}}$ orbital $\Delta_{\mathbf{i} d_{3 z^{2}-r^{2}}} / \Delta_{d_{3 z^{2}-r^{2}}^{0}}^{0}$ relative to its value in the clean system. For all results in this figure, $V_{d_{3 z^{2}-r^{2}}}=0.7 \mathrm{eV}$ and $\mu=d_{3 z^{2}-r^{2}}$ orbital.

both $T_{c}$ and $T_{m}$ seen in Fig. 2(b) appears in excellent overall agreement with the experimental results shown in Fig. 1(d). A likely explanation why the more correlated La-1111 system exhibits a smaller induced magnetic phase (compared to Sm-1111) is that larger correlations also act to more effectively screen the nonmagnetic disorder as explained in detail in the Supplemental Material [31].

We stress that the unusual disorder effects discussed in this work are not a peculiarity of certain FeSC materials, 
but general effects expected to exist in multiband correlated superconductors. For other materials close to a magnetic instability magnetic disorder should exhibit a similar aggressive $T_{c}$-suppression rate. Likewise, when nonmagnetic disorder leads to large enough LDOS enhancements of orbitals that do not dominate the spectral weight near the Fermi level, a disorder-induced coexistence phase of magnetism and superconductivity is expected to occur. Importantly, our findings also serve as a warning to draw strong conclusions about the pairing symmetry based on $T_{c}$-suppression rates of unconventional correlated systems without detailed theoretical modeling beyond conventional AG theory.

We thank P. Carretta, M. H. Christensen, F. Hammerath, P. J. Hirschfeld, D. Inosov, A. Kreisel, M. Moroni, R. di Renzi, S. Sanna, and D. J. Scalapino for useful discussions. M. N. G. and B.M. A, acknowledge support from Lundbeckfond (Grant No. A9318). F. B. acknowledges partial support from PRIN Grant No. 2012X3YFZ2-004 and from FP7-EU project SUPER-IRON (No. 283204).

[1] A. V. Balatsky, I. Vekhter, and J.-X. Zhu, Rev. Mod. Phys. 78, 373 (2006).

[2] Ø. Fischer, M. Kugler, I. Maggio-Aprile, and C. Berthod, Rev. Mod. Phys. 79, 353 (2007).

[3] H. Alloul, J. Bobroff, M. Gabay, and P. J. Hirschfeld, Rev. Mod. Phys. 81, 45 (2009).

[4] J. E. Hoffman, Rep. Prog. Phys. 74, 124513 (2011).

[5] S. Grothe, S. Chi, P. Dosanjh, R. Liang, W. N. Hardy, S. A. Burke, D. A. Bonn, and Y. Pennec, Phys. Rev. B 86, 174503 (2012).

[6] H. Yang, Z. Wang, D. Fang, S. Li, T. Kariyado, G. Chen, M. Ogata, T. Das, A. V. Balatsky, and H.-H. Wen, Phys. Rev. B 86, 214512 (2012).

[7] S. Chi et al., Phys. Rev. B 89, 104522 (2014).

[8] J.-X. Yin et al., Nat. Phys. 11, 543 (2015).

[9] A. P. Dioguardi et al., Phys. Rev. Lett. 111, 207201 (2013).

[10] X. Lu et al., Phys. Rev. B 90, 024509 (2014).

[11] S. Sanna, P. Carretta, P. Bonfá, G. Prando, G. Allodi, R. De Renzi, T. Shiroka, G. Lamura, A. Martinelli, and M. Putti, Phys. Rev. Lett. 107, 227003 (2011).

[12] S. Sanna, P. Carretta, R. De Renzi, G. Prando, P. Bonfá, M. Mazzani, G. Lamura, T. Shiroka, Y. Kobayashi, and M. Sato, Phys. Rev. B 87, 134518 (2013).

[13] Y. Bang, H.-Y. Choi, and H. Won, Phys. Rev. B 79, 054529 (2009).

[14] S. Onari and H. Kontani, Phys. Rev. Lett. 103, 177001 (2009).

[15] T. Kariyado and M. Ogata, J. Phys. Soc. Jpn. 79, 083704 (2010).

[16] D. V. Efremov, M. M. Korshunov, O. V. Dolgov, A. A. Golubov, and P. J. Hirschfeld, Phys. Rev. B 84, 180512 (2011).

[17] M. N. Gastiasoro, P. J. Hirschfeld, and B. M. Andersen, Phys. Rev. B 88, 220509(R) (2013).
[18] M. M. Korshunov, D. V. Efremov, A. A. Golubov, and O. V. Dolgov, Phys. Rev. B 90, 134517 (2014).

[19] A. A. Abrikosov and L. P. Gorkov, Zh. Eksp. Teor. Fiz. 39, 1781 (1960). Sov. Phys. JETP 12, 1243 (1961).

[20] V. Ambegaokar and A. Griffin, Phys. Rev. 137, A1151 (1965).

[21] T. V. Ramakrishnan and C. M. Varma, Phys. Rev. B 24, A137 (1981).

[22] B. M. Andersen and P. J. Hirschfeld, Phys. Rev. Lett. 100, 257003 (2008).

[23] A. Garg, M. Randeria, and N. Trivedi, Nat. Phys. 4, 762 (2008)

[24] N. Fukushima, C.-P. Chou, and T. K. Lee, Phys. Rev. B 79, 184510 (2009).

[25] A. B. Vorontsov, Ar. Abanov, M. G. Vavilov, and A. V. Chubukov, Phys. Rev. B 81, 012508 (2010).

[26] G. G. Guzmán-Verri, A. Shekhter, and C. M. Varma, Europhys. Lett. 103, 27003 (2013).

[27] S. Tang, V. Dobrosavljević, and E. Miranda, Phys. Rev. B 93, 195109 (2016).

[28] C. Castellani, C. R. Natoli, and J. Ranninger, Phys. Rev. B 18, 4945 (1978).

[29] A. M. Oles, Phys. Rev. B 28, 327 (1983).

[30] H. Ikeda, R. Arita, and J. Kunes, Phys. Rev. B 81, 054502 (2010).

[31] See Supplemental Material at http://link.aps.org/ supplemental/10.1103/PhysRevLett.117.257002 which includes Refs. [32-40], for computational details, parameter dependence, and an extended discussion of results.

[32] K. Kuroki, H. Usui, S. Onari, R. Arita, and H. Aoki, Phys. Rev. B 79, 224511 (2009).

[33] P. Lederer and D. L. Mills, Phys. Rev. 165, 837 (1968).

[34] D. Singh, Phys. Rev. B 43, 6388 (1991).

[35] E. Sjöstedt, L Nordström, and D. Singh, Solid State Commun. 114, 15 (2000).

[36] P. Blaha, K. Schwarz, G. Madsen, D. Kvasnicka, and J. Luitz, WIEN2k, An Augmented Plane Wave+Local Orbitals Program for Calculating Crystal Properties (Karlheinz Schwarz, Techn. Universität Wien, Austria, 2001).

[37] J. P. Perdew, A. Ruzsinszky, G. I. Csonka, O. A. Vydrov, G. E. Scuseria, L. A. Constantin, X. Zhou, and K. Burke, Phys. Rev. Lett. 100, 136406 (2008).

[38] C. Hess, A. Kondrat, A. Narduzzo, J. E. Hamann-Borrero, R. Klingeler, J. Werner, G. Behr, and B. Büchner, Europhys. Lett. 87, 17005 (2009).

[39] S. Sanna et al., Phys. Rev. B 82, 060508 (2010).

[40] B. P. P. Mallett, Yu. G. Pashkevic, A. Gusev, T. Wolf, and C. Bernhard, Europhys. Lett. 111, 57001 (2015).

[41] M. Sato, Y. Kobayashi, S. C. Lee, H. Takahashi, E. Satomi, and Y. Miura, J. Phys. Soc. Jpn. 79, 014710 (2010).

[42] F. Hammerath, P. Bonfá, S. Sanna, G. Prando, R. De Renzi, Y. Kobayashi, M. Sato, and P. Carretta, Phys. Rev. B 89, 134503 (2014).

[43] M. Moroni et al. (unpublished).

[44] S. J. Singh, J. Shimoyama, A. Yamamoto, H. Ogino, and K. Kishio, Physica C (Amsterdam) 494, 57 (2013).

[45] M. N. Gastiasoro and B. M. Andersen, Phys. Rev. Lett. 113, 067002 (2014)

[46] E. Satomi, S. C. Lee, Y. Kobayashi, and M. Sato, J. Phys. Soc. Jpn. 79, 094702 (2010). 
[47] K. Nakamura, R. Arita, and H. Ikeda, Phys. Rev. B 83, 144512 (2011).

[48] M. N. Gastiasoro and B. M. Andersen, J. Supercond. Novel Magn. 28, 1321 (2015).

[49] B. M. Andersen, P. J. Hirschfeld, A. P. Kampf, and M. Schmid, Phys. Rev. Lett. 99, 147002 (2007).
[50] F. Hammerath, S.-L. Drechsler, H.-J. Grafe, G. Lang, G. Fuchs, G. Behr, I. Eremin, M. M. Korshunov, and B. Büchner, Phys. Rev. B 81, 140504(R) (2010).

[51] K. Kikoin and S.-L. Drechsler, J. Magn. Magn. Mater. 324, 3471 (2012). 